\title{
The formation of intermediate mass black holes
}

\author{
Simon F. Portegies Zwart \\ Massachusetts Institute of Technology, Cambridge, MA 02139, USA, \\ Hubble Fellow
}

\begin{abstract}
We study the growth rate of stars in dense star clusters by stellar collisions. Our analytic calculations are in good agreement with direct $N$-body simulations with up to 65536 stars performed on the GRAPE family of computers. We find that star clusters with a half mass relaxation time $\lesssim 20 \mathrm{Myr}$ are dominated by stellar collisions. The first collision occurs at the moment of core collapse. The collision dominated phase last until the cluster dissolves in the tidal field of the Galaxy or mass loss by stellar evolution arrests core collapse. The majority of collisions occur with the same star resulting in the uncontrolled growth of a super massive object. This object can grow up to $\sim 0.08 \%$ of the mass of the entire star cluster. This mass ratio is comparable to the ratio of the mass of Galactic bulges to their central black hole. Star clusters which are older than about $4 \mathrm{Myr}$ and with a half mass relaxation time $\lesssim 80 \mathrm{Myr}$ are expected to contain the remnant of a phase of uncontrolled growth in their cores.
\end{abstract}

\section{Introduction}

Using Chandra, Matsumoto \& Tsuru (2001) recently discovered nine bright Xray sources in the irregular galaxy M82. Their brightest source (No. 7 in their Table 1) has a luminosity of $9 \times 10^{40} \mathrm{erg} \mathrm{s}^{-1}$, which corresponds to an Eddington luminosity of $\mathrm{a} \sim 700 \mathrm{M}_{\odot}$ compact object. A follow-up with Chandra in 2001 (Matsumoto \& Tsuru) revealed that the source had varied by more than an order of magnitude on a time scale of a few weeks. They concluded that the bright X-ray source is therefore probably a single black hole with a mass of at least $700 \mathrm{M}_{\odot}$.

An optical follow-up in the infrared ( $\mathrm{J}, \mathrm{H}$, and $\mathrm{K}^{\prime}$-bands) with the CISCO instrument on the giant SUBARU telescope revealed a star cluster with an estimated mass of a few $10^{6} \mathrm{M}_{\odot}$ on a position consistent with the X-ray location of the intermediate mass black hole (Harashima, et al. 2001). This star cluster appears to be very young ( $\lesssim 10 \mathrm{Myr}$ ) as it is extremely blue and expanding shells of molecular gas were discovered in its surrounding (Matsushita et al. 2000), typical for a star forming region. Matsushita et al (2000) estimate that the environment has an age of a few million years. 
Many more unusually bright X-ray point sources are discovered in the Antenna Galaxy (NGC 4038/4039) by Fabbiano et al. (2001) with Chandra. They conclude that these sources may be $\gtrsim 100 \mathrm{M}_{\odot}$ accreting black holes. Being an interacting pair of Galaxies, the Antennae are full of young star clusters with characteristics similar to those found in M82 (Mengel et al. 2001). It is not clear yet whether the X-ray sources and the Antennaes' star clusters are associated.

In this paper we study the possibility of forming a massive compact object in a star cluster, hereby assuming that the bright $\mathrm{X}$-ray sources are indeed intermediate mass black holes, as is suggested by Matsumoto \& Tsuru (2001). In fact, as explained below, it is quite natural to expect a $\sim 10^{3} \mathrm{M}_{\odot}$ black hole in a million $\mathrm{M}_{\odot}$ star cluster.

\section{Uncontrolled growth of a massive object in a dense star cluster}

\subsection{Core collapse and the first collision}

A star cluster is a self gravitating group of stars. As long as stellar evolution is relatively unimportant the dynamical evolution of a cluster is dominated by two body relaxation, with characteristic time scale (Spitzer, 1987)

$$
t_{\mathrm{rlx}}=\left(\frac{r^{3}}{G M}\right)^{1 / 2} \frac{N}{8 \ln \Lambda}
$$

Here $G$ is the gravitational constant, $M$ is the total mass of the cluster, $N \equiv$ $M /\langle m\rangle$ is the number of stars and $r$ is the characteristic radius of the cluster. The coulomb $\operatorname{logarithm} \ln \Lambda \simeq \ln (0.4 N)=\mathrm{O}(10)$. In convenient units the two body relaxation time becomes

$$
t_{\mathrm{rlx}} \simeq 1.9[\mathrm{Myr}]\left(\frac{r}{[\mathrm{pc}]}\right)^{3 / 2}\left(\frac{M}{\left[\mathrm{M}_{\odot}\right]}\right)^{1 / 2}\left(\frac{\left[\mathrm{M}_{\odot}\right]}{\langle m\rangle}\right)(\ln \Lambda)^{-1}
$$

The dynamical evolution of the star cluster drives it into a state of core collapse (Bettwieser \& Sugimoto 1984). In an isolated cluster where all stars have the same mass core collapse happens at about $t_{\mathrm{cc}} \simeq 15 t_{\mathrm{rlx}}$ (Cohn 1980).

Realistic cluster stars, however, have a range in masses generally from $m_{\min } \simeq 0.1 \mathrm{M}_{\odot}$ to $m_{\max } \simeq 100 \mathrm{M}_{\odot}$ and a mean mass $\langle m\rangle \sim 0.35 \mathrm{M}_{\odot}$ (Salpter 1935 ) to about $0.65 \mathrm{M}_{\odot}$ (Scalo 1986). In the early evolution of the cluster, massive stars sink to the cluster center by the interaction with other stars. A star with mass $m$ sinks from an initial distance $r_{i}$ to the cluster center in a dynamical friction time scale (Binney \& Tremaine 1987)

$$
t_{\mathrm{df}} \simeq 1.2 \frac{v}{G m \ln \Lambda} \frac{r^{2}}{r_{i}^{1 / 2}}
$$

Here $v$ is the velocity dispersion of the cluster stars. We can rewrite Eq. 3 in terms of $t_{\mathrm{rlx}}$

$$
t_{\mathrm{df}} \simeq \frac{\langle m\rangle}{m} t_{\mathrm{rlx}}
$$


Here we assumed that the star with mass $m$ was born at distance $r$ from the cluster center.

In a multi mass system core collapse is initiated by the time it takes for the most massive stars to reach the cluster center. This process takes place on a dynamical friction time scale (Eq. 4). In a star cluster where most massive stars are $\sim 100\langle m\rangle$ core collapse occurs at about

$$
t_{\mathrm{cc}}=15 t_{\mathrm{df}}\left(m_{\max }=100\langle m\rangle\right) \simeq 0.15 t_{\mathrm{rlx}}
$$

This number, of course, it taken in the limit where stellar evolution is unimportant, i.e.: where stellar mass loss is negligible and the most massive stars survive until they reach the cluster center.

The collapse of the cluster core may initiate physical collisions between stars. The product of the first collision is likely to be among the most massive stars, which is in the cluster core. This star is therefore likely to experience subsequent collisions, resulting in a collision runaway (see Portegies Zwart et al. 1999).

The maximum mass that can be grown in a dense star cluster if all collisions happen with the same star is

$$
\frac{d m_{\mathrm{r}}}{d t}=\mathcal{N}_{\text {coll }}\langle m\rangle_{\text {coll }}
$$

Here $\mathcal{N}_{\text {coll }}$ and $\langle m\rangle_{\text {coll }}$ are the average collision rate and the average mass increase per collision.

\subsection{The collision rate $\mathcal{N}_{\text {coll }}$}

The change in total energy of a star cluster during one half mass relaxation time is of the order of $10 \%$, regardless of the number of stars or of the structure of the star cluster (Goodman 1987). Following Makino \& Hut (1990) this energy is solely produced by binary heating; partly in the form of scattering products which remain bound to the system and, partially in the form of potential energy removed from the system by scattering products which escape from the system (Hut \& Inagaki 1985). Makino \& Hut (1990) argue, based on an equal mass system, that a binary deposits of the order of $10^{2} \mathrm{kT}$ in binary single star scattering. This term originates from the minimum binding energy of a binary that can eject itself following a strong encounter. The formation rate of binaries via three-body encounters is then

$$
n_{\mathrm{bf}} \simeq 10^{-3} \frac{N}{t_{\mathrm{rlx}}}
$$

The above argument is based on an analysis performed for a cluster with equal mass points. In a star cluster with a range in stellar masses, binaries generally form from stars which are more massive than average. After repeated exchange interactions the binary consists of two of the most massive stars in the cluster. Conservation of linear momentum during encounters with lower mass incoming stars makes it harder to eject the binary, as it only receives a relatively small recoil velocity. The binary has therefore to be much harder 
$\gtrsim 10^{3} k \mathrm{~T}$ before it can eject itself following a strong encounter with another star (see Portegies Zwart \& McMillan 2000).

Taking the sizes of stars into account it is then quite likely that such a hard binary experiences a collision rather than being ejected. An strong encounter between a single star and a hard binary generally results in a resonant interaction. Three stars remain in resonance until at least one of the stars has escaped or a collision reduces the three body system to a stable binary. For harder binaries it becomes increasingly more likely that a collision occurs instead of ejection (Pokman, Portegies Zwart \& Rasio 2001, in preparation). In the calculations of Portegies Zwart et al. (1999) most binaries experience a collision at a binding energy of about $10^{2} k T$, which is smaller than the binding energy required for the ejection of the binary. The collision rate per half mass relaxation time is then comparable to the three body binary formation rate

$$
\mathcal{N}_{\text {coll }} \simeq 10^{-3} f_{\text {tc }} \frac{N}{t_{\text {rlx }}} .
$$

Here we introduce $f_{\text {tc }} \lesssim 1$ the fraction of dynamically formed binaries that leads to a collision. Note that Eq. 8 is only valid in the limit where stellar evolution is relatively unimportant.

The most massive star in the cluster is typically member of the interacting binary and therefore dominates the collision rate. Subsequent collision causes the runaway to grow in mass, making it progressively harder to eject the star from the cluster. The star which experiences the first collision is therefore likely to keep participating in subsequent collisions. The majority of collisions then involve one selected object; the runaway merger. This object is generally selected by its high mass and by its proximity to the cluster center.

\subsection{The average mass increase per collision}

The average mass increase per collision depends on the characteristics the mass function in the cluster core. A lower limit for stars which participate in collisions can be derived from the amount of mass segregation in the cluster. Inverting Eq. 4 results in an estimate for the minimum mass of a star which has reached the cluster core in time $t$ due to dynamical friction

$$
m_{\mathrm{df}}=1.9\left[\mathrm{M}_{\odot}\right]\left(\frac{[\mathrm{Myr}]}{t}\right)\left(\frac{r}{[\mathrm{pc}]}\right)^{3 / 2}\left(\frac{M}{\left[\mathrm{M}_{\odot}\right]}\right)^{1 / 2}(\ln \Lambda)^{-1}
$$

Dynamical friction causes the mass function in the cluster core to becomes flatter with time, since the time scale at which a star with mass $m$ segregates to the cluster core is inversely proportional to $m$ (see Eq. 4). A cluster with a Salpeter $\left(d N(m) \propto m^{-2.35} d m\right)$ initial mass function will have a flatter mass function $\left(d N(m) \propto m^{-1.35} d m\right)$ in the core for stars more massive than $m_{\mathrm{df}}$. The mass function for the lower mass stars is not so strongly affected (see Fregeaux, Joshi, Portegies Zwart \& Rasio 2001, in preparation). Integrating the mass segregated mass function from a minimum of $m_{\mathrm{df}}$ (we ignore the upper limit) results in the mean mass in the core of

$$
\langle m\rangle_{\mathrm{coll}} \simeq 3.9 m_{\mathrm{df}}
$$


For clarity we ignored the stars with a mass $<m_{\mathrm{df}}$, which are dynamically not very important (Fregeaux et al. 2001)

Substitution of the relaxation time (Eq.1) into Eq. 9 and Eq. 10 results in a mass increase per collisions of

$$
\langle m\rangle_{\mathrm{coll}} \simeq 3.9 \frac{t_{\mathrm{rlx}}}{t}\langle m\rangle \ln \Lambda
$$

\subsection{Lifetime of a cluster in a tidal field}

A star cluster embedded in an external potential evaporates quickly. Portegies Zwart et al (2001a) studies the evolution of young compact star clusters within $\sim 200$ pc of the Galactic center. Calculations are performed using direct $N$-body integration on the GRAPE-4, including the effects of both stellar and binary evolution and the external influence of the Galaxy. The mass of their model clusters decreases almost linear with time as

$$
M=M_{0}\left(1-\frac{t}{t_{\mathrm{disr}}}\right) \text {. }
$$

Here $M_{0}$ is the mass of the star cluster at birth and $t_{\text {disr }}$ is the clusters' disruption time. Portegies Zwart et al (2001a) find $t_{\text {disr }} \simeq 0.3 t_{\text {trx }}$, with $t_{\text {trx }}$ is the relaxation time at the tidal radius $\left(t_{\mathrm{trx}}>t_{\mathrm{rlx}}\right)$.

Adopting $M=N\langle m\rangle$ throughout the cluster lifetime we can rewrite Eq. 12 in terms of the number of stars in the cluster. Substitution of Eqs. 16 and 11 in Eq. 6 gives

$$
\begin{aligned}
\frac{d m_{\mathrm{r}}}{d t} & =8 \times 10^{-4} \frac{N\langle m\rangle \ln \Lambda}{t} \\
& =8 \times 10^{-4} M_{0} \ln \Lambda\left(\frac{1}{t}-\frac{1}{t_{\text {disr }}}\right)
\end{aligned}
$$

integration from $t=t_{\mathrm{cc}}$ to $t=t_{\mathrm{disr}}$ results in

$$
m_{\mathrm{r}}=m_{\text {seed }}+8 \times 10^{-4} M_{0} \ln \Lambda\left[\ln \left(\frac{t_{\mathrm{disr}}}{t_{\mathrm{cc}}}\right)+\frac{t_{\mathrm{cc}}}{t_{\mathrm{disr}}}-1\right]
$$

Here $m_{\text {seed }}$ is the seed mass of the star which initiates the uncontrolled growth and is likely to be one of the most massive stars in the cluster. Since $t_{\mathrm{cc}} \simeq 0.15 t_{\mathrm{rlx}}$ and $t_{\text {disr }} \simeq 0.3 t_{\text {trx }}$ Eq 14 reduces to

$$
m_{\mathrm{r}}=m_{\text {seed }}+8 \times 10^{-4} M_{0} \gamma \ln \Lambda
$$

Here the $\gamma \simeq \ln t_{\mathrm{trx}} / t_{\mathrm{rlx}}+0.5 t_{\mathrm{rlx}} / t_{\mathrm{trx}}-0.3=\mathrm{O}(1)$.

\section{Results of $N$-body simulations}

The development of the GRAPE family of special purpose computers makes it relatively straight forward to test and tune the above model with $N$-body calculations. Portegies Zwart et al. (2001 in preparation) summarize the results of a larger series of detailed $\mathrm{N}$-body calculations to study core collapse and the 
collision rate in dense star clusters with up to 65536 stars. Their simulations were performed using the starlab software environment (see Portegies Zwart et al. 2001b) running on the GRAPE-6 (Makino \& Ebisuzaki 1997). To support their findings further we perform a series of simulations with $10^{4}$ stars with the same software and hardware.

\subsection{Core collapse}

In out isolated star clusters (three calculations) with $10^{4}$ single points with the same mass in a Plummer distribution core collapse occurs at $t_{\mathrm{cc}} \simeq 15.2 \pm 0.1 t_{\mathrm{rlx}}$. This result is consistent with earlier calculations of e.q., Cohn (1980) and Makino (1996). Doubling the mass of $20 \%$ of the stars reduced the core collapse time to $t_{\mathrm{cc}} \simeq 7.2 t_{\mathrm{rlx}}$. Making $20 \%$ of the stars 10 and 100 times more massive reduces the time of core collapse further to $t_{\mathrm{cc}} \simeq 1.4 t_{\mathrm{rlx}}$ and $t_{\mathrm{cc}} \simeq 0.16 t_{\mathrm{rlx}}$, respectively.

The more realistic models of Portegies Zwart et al. (1999) with 6144 and 12288 single stars taken from a Scalo (1986) initial mass function include mass loss from stellar evolution. Core collapse in their models occurred at $t_{\mathrm{cc}} \simeq$ $0.19 \pm 0.08 t_{\mathrm{rlx}}$. The slightly later collapse compared to the model with $10^{4}$ point masses may be attributed to stellar mass loss, which tends to delay core collapse.

\subsection{Collision rate}

Relaxing the assumption of point masses to stellar size introduced collision in the models. In all calculations the first collisions occur near the time of core collapse. When stars are given an unrealistically larger size (100 times larger than at zero-age) the first collision occur slightly (about $5 \%$ ) before deep core collapse.

The first star to experience a collision is generally one of the most massive stars in the cluster; this star may then become the designated target for further collisions. In models with an initial relaxation time greater than about $30 \mathrm{Myr}$ the designated target explodes in a supernova before it experiences runaway growth. The collision rate in these clusters is considerable smaller than for clusters with a smaller relaxation time (see Fig. 1). This is quite consistent with our earlier estimates. The disruption of the cluster generally terminates the uncontrolled growth.

The number of collisions in the simulation of Portegies Zwart et al. (2001, in preparation) ranges from 0 to 24 for star clusters with up to 65536 stars (see their table 2). Fig. 1 gives the collision rate $\mathcal{N}_{\text {coll }}$ per star per million years as a function of the initial relaxation time. The solid line in Fig. 1 a fit to the measurements and can be described with

$$
\mathcal{N}_{\text {coll }}=2.2 \times 10^{-4} \frac{N}{t_{\text {rlx }}} .
$$

The increase in mass per collision of the models of Portegies Zwart et al. $(1999 ; 2001)$ is consistent (within a factor of two) with the results of Eq. 11. In figure 2 we present the maximum mass of the runaway collision product as function of the initial mass of the star cluster. The results are consistent with the theoretical model presented in Eq. 14. 


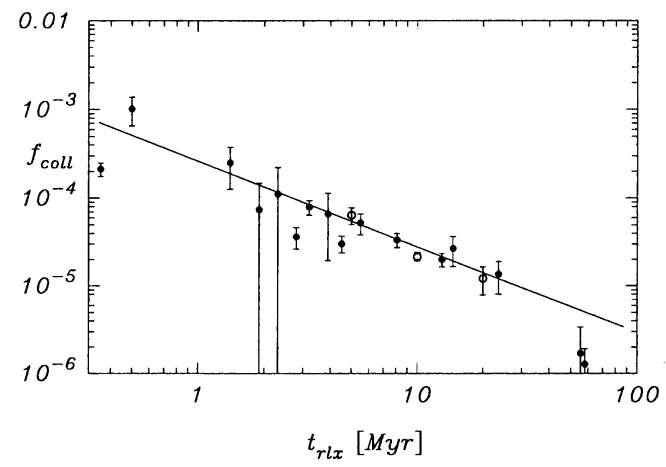

Figure 1. Collision rate $f_{\text {coll }}=N_{\text {coll }} / N t_{\text {last }}$ as function of the initial relaxation time for all models of Portegies Zwart et al 2001, see their table 2. The open circles give the results of systems which are isolated from the Galactic potential (see Portegies Zwart et al 1999). Vertical bars represent Poissonian one- $\sigma$ errors. The solid line is a least squares fit to the data (see Eq. 16). The strong reduction in the collision rate for cluster with an initial relaxation time $t_{\mathrm{rlx}} \gtrsim 30 \mathrm{Myr}$ is probably real.

\section{Discussion}

Early core collapse in dense star clusters may initiate a phase of uncontrolled growth. This leads to an object of $\sim 0.08 \%$ of the total cluster mass. We tentatively ignore the state of this object, which could be a black hole or a star. If the object is a Helium or Hydrogen burning star, it may collapse into a compact object when it exhausts its central fuel. The amount of mass lost in the supernova explosion and whether the compact object receives a velocity kick are important for the further evolution of the collision runaway. A detailed parameter study of the details concerning the supernova are beyond the scope of this paper. The basis of our analysis, however, is simple and robust to quite substantial perturbations.

We will now discuss the circumstances for which uncontrolled growth is prevented or terminated at an early stage. At the end of this section we briefly discuss the application of uncontrolled growth to the formation of super massive black holes in the bulges of galaxies.

\subsection{Early termination of the collision runaway}

Early termination of uncontrolled growth occurs when stellar mass loss starts to drive the expansion of the star cluster or when the star cluster is disrupted by external influences.

A star cluster in orbit around the Galactic center is subject to dynamical friction, much in the same way as dynamical friction drives the massive stars to the cluster center. This causes the cluster to spiral to the Galactic center, where 
it is destroyed (see Gerhard 2001). However, since the potential of the Galaxy is somewhat different than the clusters internal potential we have to re-derive the dynamical friction time scale.

The frictional drag force due to dynamical friction is (equation [7-26] in Binney \& Tremaine, 1987)

$$
F=\frac{4 \pi \ln \Lambda G^{2} M^{2} \rho_{G}(r)}{v_{\mathrm{G}}{ }^{2}}\left[\operatorname{erf}(X)-\frac{2 X}{\sqrt{\pi}} e^{-X^{2}}\right]^{-1},
$$

Here erf is the error function and $X \equiv v_{\mathrm{G}} / \sqrt{2} \sigma \simeq 0.5$, with $\sigma$ is the onedimensional velocity dispersion of the stars around the Galactic center. Evaluating the right-hand side with $X \simeq 0.5$ results in $\operatorname{erf}(X)-\frac{2 X}{\sqrt{\pi}} \exp \left(-X^{2}\right) \simeq 0.1$.

Substitution of the mass of the Galaxy within the clusters' orbit at a distance $R(\lesssim 200 \mathrm{pc}$ ) from Mezger et al. (1999)

$$
M_{\mathrm{G}}(R)=4.25 \times 10^{6}\left(\frac{R}{[\mathrm{pc}]}\right)^{1.2}\left[\mathrm{M}_{\odot}\right]
$$

and its derivative, local Galactic density (see Portegies Zwart et al. 2001)

$$
\rho_{G} \simeq 4.06 \times 10^{5} R_{\mathrm{G}}^{-1.8} \mathrm{M}_{\odot} \mathrm{pc}^{-3}
$$

in Eq. 17 results in

$$
F=-6.8 \times 10^{16} \frac{\ln \Lambda G^{2} M}{v_{\mathrm{G}}^{2} R^{0.8}} .
$$

Following Binney \& Tremaine (1987), assuming that angular momentum is conserved $\left(L=R v_{\mathrm{G}}\right)$ and that $d L / d t=F R / M$ we can integrate Eq. 20 with respect to time, which results in

$$
\begin{aligned}
t_{\mathrm{gdf}} & \simeq 8.5 \times 10^{-18} \frac{v_{\mathrm{G}}^{3} R^{1.8}}{\ln \Lambda G^{2} M} \\
& \simeq 3.6 \times 10^{3}\left(\frac{R}{1 \mathrm{pc}}\right)^{1.8}\left(\frac{v_{\mathrm{G}}}{\mathrm{kms}^{-1}}\right)^{3}\left(\frac{\mathrm{M}_{\odot}}{M}\right)[\mathrm{yr}]
\end{aligned}
$$

For clarity we assumed $\ln \Lambda=16$.

Substitution of Eqs. 18 and $v_{\mathrm{G}}{ }^{2}=G M_{\mathrm{G}} / R$ in Eq. 21 results in the time scale for dynamical friction

$$
t_{\mathrm{gdf}} \simeq 8700\left(\frac{R}{1 \mathrm{pc}}\right)^{2.1}\left(\frac{\mathrm{M}_{\odot}}{M}\right)[\mathrm{Myr}]
$$

For clusters which segregate in the Galactic center before they dissipate the maximum mass of the uncontrolled growing star can be calculated by substitution of $t_{\mathrm{disr}}=t_{\mathrm{gdf}}$ in Eq. 14. This expression then becomes a function of

$$
t_{\mathrm{gdf}} / t_{\mathrm{cc}} \simeq 7.3 \times 10^{4} \frac{R^{2.1}}{(r M)^{3 / 2}}
$$

Equating $t_{\mathrm{gdf}}=t_{\mathrm{cc}}$ results in a maximum distance to the Galactic center for which core collapse occurs $R \gtrsim 0.05(r M)^{0.71}$. 


\subsection{Prevention of the runaway}

Uncontrolled growth can only occur when stellar evolution is relatively unimportant compared to the dynamical evolution of the cluster. Stellar mass loss has the tendency to heat the cluster, by loss of potential energy. This easily reverses a collapsing core into an expanding core. Especially if one bears in mind that the most massive stars, which dominate the dynamics of the cluster core are also the first stars to lose a lot of mass in a stellar wind or a supernova. The prevention of core collapse then also prevents the initiation of the first collisions and a reversal of core collapse terminates the collision runaway.

As a rule of thumb we argue that uncontrolled growth can be prevented when the time scale for the most massive stars to segregate to the cluster center exceeds the lifetime of that star.

The main-sequence lifetime for stars more massive than $\gtrsim 30 \mathrm{M}_{\odot}$ is a rather flat function of mass $t_{\mathrm{ms}} \simeq 21 \mathrm{~m}^{-0.42} \mathrm{Myr}$. The time scale for dynamical friction, on the other hand $t_{\mathrm{df}} \simeq t_{\mathrm{rlx}}\langle m\rangle m^{-1}$. The star cluster has therefore to experience core collapse before the most massive star reaches the cluster center, i.e., $t_{\mathrm{cc}} \simeq 0.15 t_{\mathrm{rlx}} \lesssim t_{\mathrm{ms}}\left(100 \mathrm{M}_{\odot}\right) \sim 3 \mathrm{Myr}$. Runaway growth can therefore be prevented in star clusters with an initial relaxation time $t_{\mathrm{rlx}} \gtrsim 20 \mathrm{Myr}$.

In Fig. 1 star clusters with an initial relaxation time $t_{\mathrm{rlx}} \gtrsim 20 \mathrm{Myr}$ still follow Eq. 16. This is a result of the limited number of stars in these simulations, which causes the most massive stars to be somewhat less massive than $100 \mathrm{M}_{\odot}$.

\subsection{Application to massive black holes}

A star cluster formed at a distance of $\lesssim 30$ pc from the Galactic center can spiral in the Galactic center by dynamical friction before it is disrupted by the tidal field of the Galaxy (see Gerhard 2001). Only the densest star clusters survive until they reach the Galactic center. These clusters are prone to uncontrolled growth and produce a massive compact object. Upon arrival in the Galactic center the star cluster dissolves and leaves its central black hole behind. The black holes deposited by other in-spiraling star clusters may then merge to form a super massive black hole. Ebisuzaki et al. (2001) propose that such an evolution may explain the presence of the central black hole in the Milky way galaxy.

One of the great advantages of the model is that it explains the bulge and the presence of a massive black hole. The mass of the star cluster and its black hole are than related in a similar way as the bulge to its massive black hole. Galactic bulges, however, are expected to have a slightly smaller ratio of bulge-mass to black-hole mass than star clusters; not all star clusters produce a black hole and not all star clusters survive until the optimum black hole mass is reached. We expect, however, that the relation between the black hole mass and that of the bulge remains valid (see Eq. 16).

Figure 2 shows the relation between the black hole mass as function of the bulge mass for Seyfert galaxies and quasars. The expression derived in sect 2 . and the results of our $N$-body calculations (sect. 3.) are also included. The solid and dashed lines (Eq. 14) fit well through the $N$-body calculations and encloses the area of the measured black hole mass-bulge masses. On the way it passes though two measured black holes, the one in M82 and the upper limit for the globular cluster M15. 


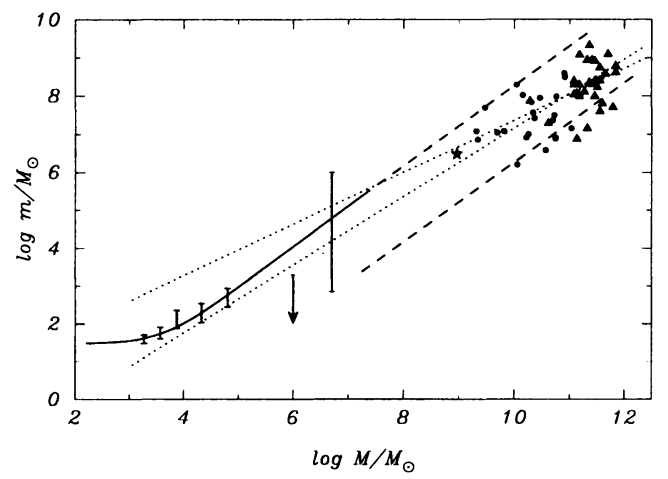

Figure 2. The mass after a period of uncontrolled growth as a function of the mass of the star cluster. The solid line gives $m_{\mathrm{r}}=30+8 \times 10^{-4} M \ln M / \mathrm{M}_{\odot}$ (see Eq. 6). For cluster with $M \gtrsim 10^{7} \mathrm{M}_{\odot}$ this line is extended as a dashed line. The bottom dashed line shows $0.01 m_{\mathrm{r}}$. The five error bars to the left give the results of the direct $N$-body calculations from Portegies Zwart et al. (2001). The data are averages of the models presented in their Table 2; from left to right: 4k stars (models KML101, KML111 and KML112), 6k (model 6k6X10), 12k (models RxW4 and 12k6X10), 14k (model KML144) and $64 \mathrm{k}$ (model N64R6r36). The down pointed arrow gives the upper limit for the mass of a compact object in the Globular cluster M15 (van der Marel, 2001) and the error bar to the right gives the mass estimate for the compact object associated with Chandra source \#7 in the irregular Galaxy of M82 (Matsumoto \& Tsuru 1999). The Milky-way is presented by the asterisk using the bulge mass from Dwek (et al. 1995) and the black hole mass from Eckart \& Genzel (1997) and Ghez (2000). Bullets and triangles (upper right) represent the bulge masses and measured black hole mass of Seyfert galaxies and Quasars, respectively (both from Wandel 1999; 2001). The dotted lines gives the range in solutions to a least squares fits to the bullets and triangles (Wandel 2001).

\section{Conclusions}

We study the uncontrolled growth of a single star in a dense star cluster using a combination of complementary approaches. Our semi-analytic analysis is supported by detailed $\mathrm{N}$-body calculations in which the effects of stellar evolution, stellar dynamics, binary evolution and the perturbing effect of a background potential are self consistently taken into account.

Star clusters with an initial relaxation time $t_{\mathrm{rlx}} \lesssim 20 \mathrm{Myr}$ experiences a phase of uncontrolled growth. In this phase a single seed star grows to a mass of about $0.08 \%$ of the total mass of the star cluster.

The first collision occurs at the moment the core of the star cluster collapses. This happens at about $0.15 t_{\mathrm{rlx}}$ but not later than about $4 \mathrm{Myr}$. The star which experiences the first collision becomes the designated target for further collisions, 
initiating the uncontrolled growth. The growth phase is terminated by 1) the disruption of the cluster in the tidal field of the Galaxy (at $t \lesssim 0.3 t_{\text {trx }}$ ) or when the core collapse is arrested by the onset of copious mass loss from its evolving stellar population (after about $20 \mathrm{Myr}$ ).

A star cluster can survive for longer than $0.3 t_{\text {trx }}$ if, for example, it was not initially filling its potential (Jacobi) surface in the tidal field of the Galaxy. (An example is $\mathrm{R} 136$, the dense star cluster in the 30 Doradus region in the Large Machelanic cloud.) Such a cluster will, as described above, go though a phase of runaway growth but recovers after stellar mass loss drives the expansion of the cluster. From an observational point of view the cluster goes through three very distinct phases: a pre collapse phase until $0.15 t_{\mathrm{rlx}}$, a phase of deep core collapse (from $0.15 t_{\mathrm{rlx}}$ to about $20 \mathrm{Myr}$ ) followed by an expansion phase leading to the disruption of the cluster. During core collapse the cluster expands causing the relaxation time to increase by a factor of 4 (see Portegies Zwart et al. 2001). A cluster in the last phase will be observable with a current relaxation time $\lesssim 80 \mathrm{Myr}$. The clearest indication of its previous phase of core collapse and runaway growth is the presence of a central compact object with a mass $\lesssim 0.08 \%$ of the initial cluster mass. The cluster may also be relatively poor in low mass compact objects (stellar mass black holes and neutron stars) as these are consumed in the runaway growth phase.

Star clusters with $t_{\mathrm{rlx}} \gtrsim 20 \mathrm{Myr}$ do not experience a phase of runaway growth as core collapse is prevented by mass loss from the most massive stars. These clusters may experience core collapse well after $\sim 100 \mathrm{Myr}$ when stellar evolution slows down again. This later core collapse, however, does not lead to a phase of uncontrolled growth. As a bonus, our simple model explains the relation between the mass of a galactic bulge and its black hole.

Acknowledgments. SPZ is grateful Jun Makino, Steve McMillan and Piet Hut for numerous discussions and he thanks the Institute for Advanced Study, Drexel University and Tokyo University for their hospitality and the use of their GRAPE-4 and GRAPE-6 hardware. This work was supported by NASA through Hubble Fellowship grant HF-01112.01-98A awarded by the Space Telescope Science Institute and by NASA ATP grants NAG5-6964 and NAG5-9264.

\section{References}

Bettwieser, E., Sugimoto, D. 1984, MNRAS, 208, 493

Binney, J., Tremaine, S. 1987, Galactic dynamics, Princeton, NJ, Princeton University Press, 1987, $747 \mathrm{p}$.

Cohn, H. 1980, ApJ, 242, 765

Dwek, E., Arendt, R. G., Hauser, M. G., Kelsall, T., Lisse, C. M., Moseley, S. H., Silverberg, R. F., Sodroski, T. J., Weiland, J. L. 1995, ApJ, 445, 716

Ebisuzaki, T., Makino, J., Tsuru, T.G., Funato, Y., Portegies Zwart, S.F., Hut, P., McMillan, S.L.W., Matsushita, S., Matsumoto, H., Kawabe, R., ApJ in press (astro-ph/0106252)

Eckart, A., Genzel, R. 1997, MNRAS, 284, 576 
Fabbiano, G., Zezas, A., Murray, S. S. 2001, ApJ, 554, 1035

Gerhard, O. 2001, ApJ, 546, L39

Ghez, A. M., Klein, B. L., Morris, M., Becklin, E. E. 1998, ApJ, 509, 678

Goodman, J. 1987, ApJ, 313, 576

Hut, P., Inagaki, S. 1985, ApJ, 298, 502

Makino, J. 1996, ApJ, 471, 796

Makino, J., Hut, P. 1990, ApJ, 365, 208

Makino, J., Taiji, M., Ebisuzaki, T., Sugimoto, D. 1997, ApJ, 480, 432

Matsumoto, H., Tsuru, T. G., Koyama, K., Awaki, H., Canizares, C. R., Kawai, N., Matsushita, S., Kawabe, R. 2001, ApJ, 547, L25

Matsushita, S., Kawabe, R., Matsumoto, H., Tsuru, T. G., Kohno, K., Morita, K., Okumura, S. K., Vila-Vilaró, B. 2000, ApJ, 545, L107

Mengel, S., Lehnert, M. D., Thatte, N., Tacconi-Garman, L. E., Genzel, R. 2001, ApJ, 550, 280

Mezger, P. G., Zylka, R., Philipp, S., Launhardt, R. 1999, A\&A, 348, 457

Portegies Zwart, S. F., Makino, J., McMillan, S. L. W., Hut, P. 1999, A\&A, 348,117

Portegies Zwart, S. F., Makino, J., McMillan, S. L. W., Hut, P. 2001a, ApJ, $546, \mathrm{~L} 101$

Portegies Zwart, S. F., McMillan, S. L. W. 2000, ApJ, 528, L17

Portegies Zwart, S. F., McMillan, S. L. W., Hut, P., Makino, J. 2001b, MNRAS, 321,199

Salpeter, E. E. 1955, ApJ, 121, 161

Scalo, J. M. 1986, Fund. of Cosm. Phys., 11, 1

Spitzer, L. 1987, Dynamical evolution of globular clusters, Princeton, NJ, Princeton University Press, 1987, 191 p.

van der Marel, R. P. 2001, in Black Holes in Binaries and Galactic Nuclei. Proceedings of the ESO Workshop held at Garching, Germany, 6-8 September 1999. Lex Kaper, Edward P. J. van den Heuvel, Patrick A. Woudt (eds.), p. 246. Springer., p. 246+

Wandel, A. 1999, ApJ, 519, L39

Wandel, A. 2001, in submitted to the ApJ, 8461 